\title{
El aprendizaje independiente y el rendimiento académico de los estudiantes universitarios
}

The independent learning and the academic performance of university students

Jaqueline Jessica Cabello Blanco', Enrique Fernando Tello Rodríguez ${ }^{1}$

\section{RESUMEN}

Objetivo: Determinar que el aprendizaje independiente incrementa el rendimiento académico de los estudiantes universitarios. Métodos: La población estuvo constituida por los estudiantes del II ciclo de la Escuela Profesional de Ingeniería Zootecnica, matriculados en el curso de matemática II, semestre académico 2015-I y 2015-II, Universidad Nacional José Faustino Sánchez Carrión, Huacho, Provincia de Huaura, Departamento de Lima. Tipo de investigación experimental, se utilizó el método comparativo, el diseño fue cuasi experimental, transversal, con tratamiento repetido; a los estudiantes del semestre académico 2015-I se les enseñó con aprendizaje formal y a los estudiantes del semestre académico 2015-II se les enseñó con el aprendizaje independiente. Para el análisis de datos se utilizó la estadística descriptiva y estadística inferencial (Kolmogorov- Smirnov y U de Mann-Whitney). Resultados: con la prueba de Kolmogorov-Smirnov se encontró que la población en estudio es de libre distribución $(r=0,01)$; así con ayuda de la prueba de contraste de hipótesis $U$ de Mann-Whitney $(r=0,01)$ se encontró que existe diferencia entre los rendimientos de los estudiantes y que la media del rendimiento académico de los estudiantes en el semestre académico 2015 II es mayor que el semestre académico 2015 I (11>8). Por otro lado el 100\% de los estudiantes respondió que el aprendizaje independiente es parte de la enseñanza aprendizaje. Asimismo, el 100\% de los estudiantes lograron establecerse metas durante el desarrollo del curso de matemática II, logrando el $85 \%$ cumplir sus metas. Además el $60 \%$ de los estudiantes refieren que han sido capaces de crear y resolver problemas fuera de clases. Conclusiones: Siendo $r=0,003$, se concluye con un nivel de significancia de $a=0,05$ que existe diferencia altamente significativa entre los grupos de aprendizaje independiente y aprendizaje formal respecto al rendimiento académico de los estudiantes, siendo mayor el rendimiento académico de los estudiantes que tuvieron un aprendizaje independiente.

Palabras clave: Aprendizaje, aprendizaje independiente, rendimiento académico.

\section{ABSTRACT}

Objective: To determine that the independent learning increases the academic performance of university students. Methods: The population in which this inquiry was conducted, was constituted by the students of the II engineering cycle zootechnics, enrolled in the course of mathematics II, academic semester 2015-I and II, National University Jose Faustino Sanchez Carrion, Huacho, Province of Huaura, department of Lima. Type of basic research, the comparative method was used, the design was a quasi experimental cross-sectional, with repeated treatment., to students in the academic semester 2015-I was taught with formal learning and the students of the academic semester 2015-II were taught with independent learning. Was used for data analysis the descriptive statistics (tables), and inferential statistics (Kolmogorov-Smirnov and $U$ deMann- Whitney). Results: With the test Kolmogorov-Smirnov it was found that the study population is for free distribution $(r=0,01)$; and with the help of the contrast test of hypothesis Mann- Whitney U-test $(r=0,003)$ found that there is a difference between the yields of the students and that the average of the academic performance of the students in the academic semester $2015 \mathrm{II}$ is greater than the academic semester $2015 \mathrm{I}(11>8)$. On the other hand the $100 \%$ of students responded that the independent learning is part of the teaching and learning. Also, the 100 per cent of the students were able to set goals during the development of the course of mathematics II, achieving $85 \%$ meet its goals. In addition $60 \%$ of students refer that have been able to create and resolve issues outside of classes. Conclusions: Being $(r=0,003)$ and $11>8$, concludes with a level of significance of $a=0.05$ that there is a highly significant difference between the independent learning groups and formal learning regarding students' academic performance, with the academic performance of students who had independent learning.

Keywords: Learning, independent learning, academic performance.

\footnotetext{
${ }^{1}$ Universidad Nacional José Faustino Sánchez Carrión. Huacho, Perú.
} 


\section{INTRODUCCIÓN}

La sociedad de hoy se caracteriza por un creciente número de investigaciones que son publicadas por internet. Nuestra Universidad Nacional José Faustino Sánchez Carrión no es ajena a ese desarrollo, en la actualidad existen muchos distractores en la atención del estudiante, redes sociales, gran cantidad de información, tecnología al alcance, los mismos que no son usados adecuadamente para su aprendizaje, por lo que se ha ido observando a lo largo de estos años que los estudiantes del curso de matemática II de la Escuela Profesional de Zootécnica presentan un bajo rendimiento académico en el área de matemática, sus aprendizajes son meramente memorísticos o repetitivos, con un creciente porcentaje de desaprobados, encontrándose en el semestre 2015 I un $4 \%$ de excelentes estudiantes, $0 \%$ de muy bueno, $13 \%$ de bueno, $30 \%$ de regulares y un $52 \%$ de pésimo. Muchas veces se escucha decir a los estudiantes ¿para qué nos va a servir la matemática?, la matemática en la formación de los estudiantes es importante ya que les ayuda a ejercitar su razonamiento, para ser creativos e innovadores, cosa indispensable para entender $y$ atender a los problemas del mundo real que se enfrentarán al termino de sus estudios, esto y otros factores (personales y sociales) asociados a la estructuración del sistema didáctico de nuestra universidad, donde los docentes están poco tiempo con sus estudiantes, cumpliendo solo las horas de clases establecidos por cada curso y sin estrategias de instrucción planificada para fomentar el aprendizaje significativo lo que lleva a los estudiantes a un bajo rendimiento en el curso de matemática. Es por esto que los estudiantes deben ser protagonistas de su aprendizaje para una formación sólida, la tarea de los docentes, en consecuencia, consiste además del tratamiento didáctico de ciertos contenidos matemáticos, es desarrollar métodos para un aprendizaje independiente y pasar de la enseñanza puramente receptiva a una enseñanza basada en el descubrimiento e independencia.

Valera (2009) menciona que el aprendizaje es un proceso en el cual la intervención social es básica; la interacción y el apoyo con otras personas, así como el contexto en que se aprende son elementos esenciales. Pero finalmente, el aprendizaje es un proceso individual en el que cada persona es quien adquiere y cambia de manera única; la construcción del conocimiento es interna y personal. Es un trabajo personal alcanzado a través de diversas estrategias.

Rué (2009) señala que el estudiante en una situación de aprendizaje formal no puede prescindir del profesor, del programa, del contexto curricular, etc., tal como puede hacerlo alguien que decida aprender algo por su cuenta al margen de todo reconocimiento y marco institucional, a la manera autodidacta. Este último sería el significado que reservaríamos para un aprendizaje independiente. La autonomía en el aprendizaje, si bien son valores del mismo proceso de formación, su despliegue y asimilación personal requieren que se haga desde el enfoque de competencias.

Valera (2009) señala que el aprendizaje independiente o autorregulado es definido como un aprendizaje activo en el que los estudiantes asumen la responsabilidad para motivarse a sí mismos y entender el material que estudian. Al desarrollar un aprendizaje independiente o autorregulado, el estudiante planea, lleva a cabo de manera autocontrolada actividades de aprendizaje y es capaz de evaluar de manera crítica los resultados que alcanza. Es una forma elevada de involucrarse en el propio proceso de aprender, en la que el mismo alumno supervisa y controla su progreso.

Así, se define al aprendizaje independiente como una metodología (forma) que empleamos como estudiantes para aprender; consiste en promover mayor autonomía y fortalecimiento de todos aquellos modos de acción que aseguren la actualización continua, con el aprendizaje independiente se delega la mayor responsabilidad del aprendizaje al estudiante, donde este aprenderá el buen uso de los recursos materiales y humano, elegir estrategias adecuadas a sus necesidades, evaluar los resultados obtenidos. Se es autónomo cuando eres capaz de gobernarte a ti mismo(a) y eres menos gobernado por los demás. La esencia de la autonomía es que las personas lleguen a ser capaces de tomar sus propias decisiones.

Con los hallazgos de esta investigación se facilitará la mejora de la enseñanzaaprendizaje del curso de Matemática II, de la Escuela Profesional de Zootécnica, dado que el aprendizaje independiente, sobre todo en la 
Educación Matemática, es un medio para desarrollar en el estudiante habilidades de independencia cognitiva, cooperación, comunicación, actitudes y formación en general. Así, la Universidad Nacional José Faustino Sánchez Carrión formará profesionales con un alto grado de independencia cognitiva, creatividad y toma de decisiones para la solución de problemas locales dado que en su proceso formativo se podrá lograr un ambiente de enseñanzaaprendizaje en el cual los estudiantes sean artífice de su formación.

Esta investigación a diferencia de Valera (2009) se realizó en estudiantes en el área de matemática, para constituirse así una metodología de enseñanza-aprendizaje con recursos que no solo desarrollen habilidades cognitivas que faciliten los cálculos sino que también sea una herramienta para la formación integral de los estudiantes.

En ese contexto, el objetivo de esta investigación es determinar que el aprendizaje independiente incrementa el rendimiento académico de los estudiantes del curso de matemática II de la Escuela Profesional de Zootécnica de la Universidad Nacional José Faustino Sánchez Carrión - 2015. Teniendo como hipótesis que el rendimiento académico es mayor entre los estudiantes que tuvieron un aprendizaje independiente comparado con los estudiantes que tuvieron un aprendizaje formal.

\section{MATERIAL Y MÉTODOS}

La población en la que se realizó la presente investigación, estuvo constituida por los 28 estudiantes del II ciclo de la Escuela Profesional de Ingeniería Zootécnica, matriculados en el curso de matemática II, del semestre académico 2015-I y 48 estudiantes del II ciclo de la Escuela de Ingeniería Zootécnica, matriculados en el curso de matemática II, del semestre académico 2015-II de la Universidad Nacional José Faustino Sánchez Carrión - Huacho, provincia de Huaura, departamento de Lima. En el semestre académico 2015-I a los estudiantes se les impartió el curso de matemática II con el aprendizaje formal de la institución, luego se les evaluó las capacidades y el conocimiento frente a lo desarrollado; en el semestre académico 2015-II a los estudiantes se les impartió talleres de como aprender a prender, esto es saber hacer y controlar el curso de matemática II para construir su conocimiento sobre el uso de estrategias y tomar decisiones de cuando y como aplicarlas, y se les evaluó las capacidades y el conocimiento frente a lo desarrollado, adicional a esto se les aplicó una encuesta para analizar el impacto del aprendizaje independiente en los estudiantes.

\section{RESULTADOS}

Según Gutiérrez (2003) para conocer la distribución de la población en estudio, se utiliza las pruebas de hipótesis de KolmogorovSmirnov donde se asume que la muestra proviene de una población con distribución normal, a lo cual se obtuvo que la población en estudio es de libre distribución, es decir no sigue una distribución normal según el estadístico de prueba Kolmogorov-Smirnov con $p<0,001$.

Conocida que la muestra es de libre distribución, se procedió a realizar la prueba de contraste de hipótesis, en este caso, la $U$ de Mann - Whitney (Berenson y Levine, 1996), donde se obtuvo una diferencia altamente significativa por ser $p=0,003$, esto explica que el ranking del rendimiento académico 2015-I es de 08 y el del rendimiento académico 2015-II es de 11 es decir hay diferencias entre el rendimiento académico 2015-I y del rendimiento académico 2015-II.

El rendimiento académico es diferente entre el aprendizaje independiente y el aprendizaje formal, siendo mayor el rendimiento académico de los estudiantes que tuvieron un aprendizaje independiente con un nivel de significancia $a=0,05$; Si bien es cierto el promedio del rendimiento académico es de 08 a 11entre los estudiantes del 2015-I y 2015-II, con un incremento del $2 \%$ en sus calificaciones, se observa que los estudiantes están en el camino de su autonomía en su aprendizaje que claro al inicio tendrán incertidumbre, dificultades y obstáculos que poco a poco serán capaces de tomar sus propias decisiones y alcanzar sus objetivos en lo que concierne a su aprendizaje y repercutiendo en su desarrollo personal. 


\section{Análisis de la encuesta}

\begin{tabular}{|c|c|}
\hline Pregunta & Respuesta en porcentaje de los estudiantes \\
\hline $\begin{array}{l}\text { ¿Considera usted que el aprendizaje independiente es parte de la } \\
\text { enseñanza aprendizaje? }\end{array}$ & $\begin{array}{l}\text { El } 100 \% \text { de los estudiantes refirieron que el aprendizaje independiente sí } \\
\text { es parte de la enseñanza aprendizaje. }\end{array}$ \\
\hline $\begin{array}{l}\text { ¿Durante el desarrollo del curso usted ha logrado establecerse metas } \\
\text { y alcanzar su meta? }\end{array}$ & $\begin{array}{l}\text { El } 100 \% \text { de los estudiantes refirieron que sí se establecieron metas } \\
\text { durante el desarrollo del curso de matemática II. } \\
\text { El } 85 \% \text { de los estudiantes lograron alcanzar sus metas. }\end{array}$ \\
\hline $\begin{array}{l}\text { ¿La universidad cuenta con un lugar apropiado para desarrollar su } \\
\text { estudio independiente? }\end{array}$ & $\begin{array}{l}\text { El } 100 \% \text { de los estudiantes refieren que la universidad no cuenta con } \\
\text { ambiente apropiado para desarrollar su estudio independiente. }\end{array}$ \\
\hline $\begin{array}{l}\text { ¿La universidad cuenta con material bibliográfico para los diferentes } \\
\text { cursos que lleva este semestre? }\end{array}$ & $\begin{array}{l}\text { El } 100 \% \text { de los estudiantes refieren que la universidad no cuenta con } \\
\text { material bibliográfico variado para el desarrollo de los diferentes cursos } \\
\text { del semestre. }\end{array}$ \\
\hline ¿Cuenta usted con material bibliográfico para el desarrollo del curso? & $\begin{array}{l}\text { El } 86 \% \text { de los estudiantes refirieron que si cuentan con material } \\
\text { bibliográfico y el } 14 \% \text { de los estudiantes no cuentan con material } \\
\text { bibliográfico para el desarrollo del curso. }\end{array}$ \\
\hline $\begin{array}{l}\text { ¿Usted se apropia de mayor conocimiento en clases o en el estudio } \\
\text { independiente? }\end{array}$ & $\begin{array}{l}\text { El } 68 \% \text { de los estudiantes refirieron que fuera de clases revisando libros y } \\
\text { reuniéndose en grupo adquieren mayor conocimiento y el } 32 \% \text { de los } \\
\text { estudiantes adquieren conocimientos mayormente en clases. }\end{array}$ \\
\hline $\begin{array}{l}\text { ¿Es capaz de crear y resolver problemas durante su estudio } \\
\text { independiente? }\end{array}$ & $\begin{array}{l}\text { El } 60 \% \text { de los estudiantes refirieron que han sido capaces de crear y } \\
\text { resolver problemas fuera de clases ¿Es capaz de crear y resolver } \\
\text { problemas durante su estudio independiente? revisando libros y } \\
\text { reuniéndose en grupo, el } 40 \% \text { de los estudiantes refieren que aún tienen } \\
\text { dificultades al momento de resolver problemas fuera de clases. }\end{array}$ \\
\hline
\end{tabular}

\section{DISCUSIÓN}

Dentro de los resultados encontrados se tiene que el rendimiento académico es diferente entre el aprendizaje independiente y el aprendizaje formal, siendo mayor el rendimiento académico de los estudiantes que tuvieron un aprendizaje independiente. Román y Herrera (2010), presentan una propuesta que concibe el trabajo independiente como proceso de dirección, a partir del cual se incide en la formación del profesional y se pone énfasis en la necesidad de cambiar las formas de dirigir el proceso formativo contemporáneo. A partir de nuestros hallazgos podemos observar que los estudiantes son protagonistas de su aprendizaje y son motivados a la adquisición de conocimientos y la explotación útil de las tecnologías de la información y las comunicaciones para una formación sólida.

Coincidimos con Román y Herrera (2010) que el aprendizaje independiente sea concebido como proceso didáctico a partir del cual se incide en la formación integral del profesional, dado que está relacionado con la meta cognición entendida como autocontrol. Por otro lado, actualmente se está incorporando el uso de la tecnología, tanto como un apoyo a la enseñanza y para sustentar el aprendizaje independiente del estudiante. Por tanto las tecnologías se incorporan al aprendizaje independiente.

El aprendizaje independiente logró en los estudiantes la capacidad de crear y resolver problemas fuera de clases, revisando libros y

obligándolos a formar grupos de trabajo, además de incrementar sus conocimientos.

\section{REFERENCIAS BIBLIOGRÁFICAS}

Berenson, M. \& Levine, D. (1996). Estadística básica en administración. 6a edición. $M$ é x i co: $\mathrm{Pr}$ e n t i c e - $\mathrm{H}$ a I I Hispanoamericana.S.A.

Gutiérrez, H. De la Vara, R. (2003). Análisis y diseño de experimentos. México D.F.: Ed. McGraw Hill.

Román-Cao, E., \& Herrera-Rodríguez, J. I. (2010). Aprendizaje centrado en el trabajo independiente. Educación y educadores, 13(1).

Rué, J. (2009). El aprendizaje autónomo en educación superior (Vol. 20). Narcea Ediciones.

Valera, M. (2009). Aprendizaje independiente y aprendizaje colaborativo en educación médica. Revista médica del Hospital General de México, 72(4), 222-227. Recuperado: 6 de noviembre del 2015. D i s p o n i b le e n : http://www.medigraphic.com/pdfs/hgral/hg-2009/hg094i.pdf

Correo electrónico: jcabello@unjfsc.edu.pe

Revisión de pares:

Recibido: 19-09-2016

Aceptado: 19-12-2016 